\title{
CIENCIAS DE LA COMPLEJIDAD VS. PENSAMIENTO COMPLEJO. CLAVES PARA UNA LECTURA CRÍTICA DEL CONCEPTO DE CIENTIFICIDAD EN CARLOS REYNOSO
}

\author{
MIGUEL RAMÓN VIGURI AXPE \\ Universidad de Deusto. Bilbao
}

\begin{abstract}
RESUMEN: El antropólogo social Carlos Reynoso ha planteado una serie de objeciones al llamado pensamiento complejo, cuyo principal representante es el pensador francés Edgar Morin. Reynoso toma como punto de referencia para realizar la crítica al pensamiento complejo el rigor conceptual de las ciencias de la complejidad. Sus acusaciones sobre el pensamiento complejo se fundamentan en el carácter discursivo de éste y en su falta de un método científico. Este artículo analiza y critica el concepto implícito de cientificidad en el pensamiento de Reynoso y propone la necesidad de articular pensamiento filosófico y metodología empírica para comprender la complejidad y sus ciencias.
\end{abstract}

PALABRAS CLAVE: Reynoso; ciencia; ciencias de la complejidad; Morin.

\section{Science of complexity vs. complex thinking \\ keys for a critical reading of the concept of scientific in Carlos Reynoso}

ABSTRACT: The social anthropologist Carlos Reynoso has raised a series of objections to the socalled complex thinking, whose main representative is the French thinker Edgar Morin. Reynoso takes as point of reference to critique complex thinking the conceptual rigor of complexity sciences. His objections against complex thinking are based on his discursive nature and his lack of a scientific method. This article analyzes and criticizes the implicit concept of scientificity in Reynoso's thinking and proposes the need to articulate philosophical thinking and empirical methodology to understand complexity and its sciences.

KEY WORDS: Reynoso; science; complexity sciences; Morin.

\section{INTRODUCCIÓN}

El antropólogo social Carlos Reynoso ${ }^{1}$ ha planteado una serie de objeciones al llamado pensamiento complejo, cuyo principal representante es el pensador francés Edgar Morin. Dicha crítica la realiza básicamente en dos de sus obras: Complejidad y $\mathrm{Caos}^{2}$, y Modelos o metáforas ${ }^{3}$.

1 El antropólogo Carlos Reynoso es Doctor en Antropología Social por la Universidad Nacional de Misiones y Profesor Regular del Departamento de Ciencias Antropológicas de la Facultad de Filosofía y Letras de la Universidad de Buenos Aires, Argentina. Allí imparte las cátedras de Teorías Antropológicas Contemporáneas y Elementos de Lingüística y Semiótica (de las cuales es adjunto y titular respectivamente), junto a diversos seminarios de grado y posgrado.

Fuente: Página principal de la web de Carlos Reynoso, http://carlosreynoso.com.ar/

2 Reynoso, C., Complejidad y Caos. Una exploración antropológica, Buenos Aires 2006.

3 Reynoso, C., Modelos o Metáforas. Crítica del paradigma de la complejidad de Edgar Morin, Buenos Aires 2009. 
En su obra Complejidad y Caos critica rigurosamente lo que él considera abusos conceptuales por parte del pensamiento complejo moriniano, aunque también incluye críticas a los planteamientos sobre la complejidad de personalidades de reconocido prestigio científico, tales como el premio Nobel Ilia Prigogine, o los biólogos chilenos Humberto Maturana y Francisco Varela ${ }^{4}$.

Carlos Reynoso toma como punto de referencia para realizar la crítica al pensamiento complejo el rigor conceptual de las ciencias de la complejidad. Sus acusaciones sobre el pensamiento complejo se fundamentan en el carácter discursivo de éste (formulado en lenguaje natural) y, por lo tanto, en la polivalencia de sus conceptos que provoca frecuentemente debates circulares que conducen a callejones sin salida.

Como aclaración inicial, diremos que el pensamiento complejo fue desarrollado por Edgar Morin en su obra El Método ${ }^{5}$. Aunque, más que propiamente un método para resolver problemas complejos, E. Morin, en dicha obra, constata la insuficiencia de un modo de pensamiento lineal y determinístico, que es el que ha caracterizado a las ciencias que se han movido dentro de un paradigma mecanicista. Es decir, que lo que intenta Morin con su obra es poner al descubierto las insuficiencias de la ciencia clásica ante una realidad que es compleja; esto es, abierta, evolutiva, nolineal y caracterizada por la estructura recursiva de las relaciones entre los diversos elementos que la integran y que da lugar a la emergencia de nuevas propiedades y elementos.

Así pues, el pensamiento complejo de E. Morin es una especie de epistemología (una nueva forma de mirar la realidad y de entender el propio conocimiento humano de dicha realidad) que funge como pedagogía de una nueva forma de pensamiento: lo que E. Morin denomina el paradigma de la complejidad. Se trata de un planteamiento genuinamente filosófico, tal y como entiende C. E. Maldonado 6 .

Las ciencias de la complejidad, por el contrario, son desarrollos teóricos con una fuerte base matemática que han nacido en el ámbito de las ciencias naturales (como es el caso de la termodinámica de los sistemas alejados de equilibrio, desarrollada por Ilia Prigogine ${ }^{7}$ ) y de las ciencias formales

4 Cfr. Maturana, H. y Varela, F., De máquinas y seres vivos, $6^{\text {a }}$ Edición, Buenos Aires 2004; The tree of knowledge: The biological roots of human understanding, Boston 1987 (hay traducción castellana: El árbol del conocimiento. Las bases biológicas del entendimiento humano, Buenos Aires 2003).

5 Morin, E., La Méthode 1. La nature de la Nature, Editions du Seuil, 1977; La Méthode 2. La vie de la Vie, Editions de Seouil, 1980; La Méthode 3. La connaissance de la Connaisance, Éditions de Seuil, 1986; La Méthode 4. Les idées, leur hábitat, leur vie, leurs moeurs, leur organisation, Editions de Seouil, 1991; La Méthode 5. L'Humanité de l'humanité. L'Identité humaine, Editions de Seouil, 2001; La Méthode 6. Éthique, Editions de Seouil, 2003. Los seis volúmenes que integran la obra están traducidos al castellano por Ediciones Cátedra.

6 Cfr. Maldonado, C. E., Visiones de la complejidad, Bogotá 2001, p. 16.

7 Cfr. Prigogine, I., Introduction to thermodynamics of irreversible proccesses, New York, 1961; La fin des certitudes: Temps, chaos et les lois de la nature, Paris, 1996. 
como la matemática (la teoría del caos - uno de cuyos pioneros fue $\mathrm{H}$. Poincaré $^{8}$ al formular el problema del tercer cuerpo-, la geometría de fractales de B. Mandelbrot ${ }^{9}$ y la teoría de catástrofes de R. Thom ${ }^{10}$, serían las más conocidas).

Estas ciencias estudian fenómenos caracterizados por no-linealidad y, por tanto, con un componente de impredictibilidad. Para ello recurren a sistemas formales que pueden implementarse algorítmicamente y plasmarse en modelos de simulación computacional.

Pues bien, la controversia entre pensamiento complejo y ciencias de la complejidad consiste en decidir cuál de estos dos sistemas de pensamiento caracteriza mejor el fenómeno de la complejidad. Y la postura de C. Reynoso es clara: el pensamiento complejo únicamente puede aportar una serie de metáforas, dado que se trata de una teoría de tipo discursivo sin incidencia en la realidad. En cambio, las ciencias de la complejidad, mediante la elaboración de modelos algorítmicos permiten ir dando respuesta a determinados problemas complejos y, por ello, permiten ir delimitando qué es eso que denominamos complejidad. Este es el planteamiento que subyace a su libro Modelos o Metáforas, cuyo título es bien expresivo respecto a dónde sitúa Reynoso la controversia.

Aparte de C. E. Maldonado y de C. Reynoso, ha habido otros autores que han criticado el pensamiento complejo y que, como señalan Leonardo Rodríguez Zoya y Paula Rodríguez Zoya en un magnífico artículo ${ }^{11}$, también han reflexionado críticamente sobre las ciencias de la complejidad, como son Rafael Pérez-Taylor ${ }^{12}$ y Rolando García ${ }^{13}$. Incluso hay otros autores, como Jorge Karel Leyva Rodríguez ${ }^{14}$, o, Juan Félix Burotto y Francisco GangaContreras ${ }^{15}$ que han dedicado a esta polémica algunos artículos en revistas especializadas. En todas esas obras encontramos importantes reflexiones acerca de la interdisciplinariedad y la naturaleza propia de la complejidad, junto a reflexiones de tipo metodológico con la finalidad de establecer una taxonomía adecuada de las distintas disciplinas que integran ese mundo de la complejidad.

8 Cfr. Poincaré, E., Science et méthode, París, 1909.

9 Cfr. Mandelbrot, B., The fractal geometry of nature, New York, 1983.

10 Cfr. Tном, R., Parábolas y catástrofes, Barcelona, 1985.

11 Cfr. Rodríguez Zoya, L. y Rodríguez Zoya, P., «El espacio controversial de los sistemas complejos», en Estudios Filosóficos, 50 (2014) 124-127.

12 Cfr. Pérez Taylor, R., Antropología y complejidad, Barcelona 2002. Y también: Anthropologías: avances en la complejidad humana, Buenos Aires 2006.

13 Cfr. García, R., Sistemas complejos. Conceptos, método y fundamentación epistemológica de la investigación interdisciplinaria, Barcelona 2006.

14 Leyva Rodríguez, J. K., «El evangelio según San Morin. Una crítica a la concepción religiosa del pensamiento complejo», en: A Parte Rei, Revista de Filosofía, 59 (2007) 1-18.

15 Burotto, J. F. y Ganga-Contreras, F., «La méthode de Morin: refutaciones y aperturas», en: Convergencia. Revista de Ciencias Sociales, México, 19, nº 60 (2012) 79-109. 
En cambio, la crítica que ha realizado Carlos Reynoso al pensamiento complejo (sobre todo al pensamiento complejo moriniano) es extensa (ha dedicado a ello dos libros y varios artículos), específica y sistemática.

C. Reynoso considera que solamente las ciencias de la complejidad están en condiciones de producir unos conceptos y un acercamiento a una Teoría General de la Complejidad que realmente cumpla con las funciones que debiera de tener una auténtica epistemología de la complejidad (que es como Edgar Morin presenta su pensamiento complejo) ${ }^{16}$. Así pues, C. Reynoso propone su crítica como una crítica científica del pensamiento complejo; es decir, una crítica con pretensión de carácter demostrativo, objetividad y validez científica ${ }^{17}$.

Finalmente, la crítica de C. Reynoso no se ciñe a determinados aspectos del pensamiento complejo, sino que pretende ser una deslegitimación de dicho pensamiento en su totalidad ${ }^{18}$.

Por estas razones, y por la importancia que tiene la epistemología del pensamiento complejo para la elaboración de pensamiento interdisciplinar (fundamental para el adecuado planteamiento y resolución de los problemas globales y complejos que aquejan a una sociedad globalizada como la nuestra), creo necesario revisar algunos de los argumentos utilizados por C. Reynoso y, sobre todo, su concepción de la ciencia (pues es en dicha concepción en la que basa su argumentación).

Esta es también la opinión de los profesores Leonardo Rodríguez Zoya y Paula Rodríguez Zoya:

16 Morin, E., «La epistemología de la complejidad», en: Gazeta de Antropología, no 20 (2004), texto 20-02.

17 «Como tantos otros, este libro se escribe desde una posición tomada, que en este caso deberé admitir que es clásicamente científica. Es la que ha producido los fundamentos teóricos, los algoritmos y los recursos computacionales concretos, aunque tal vez no sea en estos tiempos la más popular en el plano mediático». Reynoso, C., Modelos o metáforas, Crítica del paradigma de la complejidad de Edgar Morin, Buenos Aires 2009, p. 20.

18 «El hecho es que el modelo moriniano elude todo tratamiento de las teorías y métodos del último cuarto de siglo en el terreno complejo y no logra retratar con fidelidad la literatura sistémica anterior. En tanto lectura científica se halla sobredeterminada por el afán de impartir premios y condenas en función de criterios sectarios que a fuerza de ser pequeños resultan consabidos, y por el empeño de Morin de constituirse en el mediador por excelencia entre cierta región de la ciencia y las humanidades como si ningún otro pensador hubiera explorado ese espacio. En la ejecución de este plan se estrella con unas ciencias duras que lo desbordan y se distrae en un despliegue enciclopédico que no guarda proporción con las destrezas especializadas requeridas en ese terreno. Su programa no sólo falla en el terreno algorítmico, como sería de esperar, sino también y sobre todo en el epistemológico. Como demostraré luego con la paciencia que haga falta, el estilo es impropio, las carencias fehacientes, los errores muchos. Empañada por estos factores, su erudición suena más ampulosa que elegante cada día que pasa y en estos tiempos de disponibilidad masiva de información su magnitud no luce tan admirable como alguna vez se creyó que era». REyNoso, C., Modelos o Metáforas. Crítica del paradigma de la complejidad de Edgar Morin, Buenos Aires 2009, 10-11. 
«Ahora bien, si se analiza con detalle el principio que sustenta la tipología de Reynoso, se observa que su propuesta no se sostiene desde el punto de vista epistemológico ni de la historia de la ciencia. Más aún, y esta es la conclusión de la argumentación que sigue, el foco controversial que instaura Reynoso no se halla, de hecho, en el dualismo teórico-empírico, sino en intentar esclarecer lo que constituye, a su juicio, una mirada «genuina», «correcta» $\mathrm{y}$ «científica» de la realidad $»^{19}$.

No me voy a centrar en responder a las acusaciones de Reynoso sobre el paradigma del pensamiento complejo porque ya ha habido otros académicos de reconocida solvencia intelectual que lo han hecho con suficiente rigor. Me refiero concretamente a José Luis Solana Ruiz ${ }^{20}$ (profesor del Departamento de Antropología, Geografía e Historia de la Universidad de Jaén, España) y a Leonardo y Paula Rodríguez Zoya (en el ya citado artículo).

El objeto central de mi crítica es el hecho de que Carlos Reynoso considere que el significado de los conceptos con los que se pretenda describir o caracterizar la complejidad, tengan que provenir exclusivamente del uso que se da a dichos conceptos en el campo de las ciencias que él llama «ciencias duras». Carlos Reynoso considera que el lenguaje científico propio de las ciencias experimentales es el único que garantiza univocidad en el significado de los conceptos, y dicha univocidad es imprescindible en ciencia.

Por ello, insisto, lo que llama mi atención en los escritos de Carlos Reynoso, y de lo que me voy a ocupar en mi análisis de la propuesta de dicho autor, no es tanto la cuestión de la complejidad (cómo se la caracterice y cómo se la entienda), cuanto cómo entiende Reynoso lo que es hacer ciencia en el marco de las ciencias de la complejidad; así como la relación que, para él, guardan las "ciencias duras» con las «ciencias blandas».

En este sentido, es interesante el comentario de los autores Leonardo y Paula Rodríguez Zoya:

«A la luz de los argumentos expuestos es preciso considerar el problema de la "demarcación". La preocupación de Maldonado y Reynoso reviven la vieja inquietud positivista acerca de un criterio de demarcación entre la ciencia y la metafísica. En este sentido, ambos autores se esfuerzan por señalar los fundamentos que permitan precisar en qué consiste una manera "genuina" y "correcta" de abordar el estudio científico de la complejidad. Maldonado aboga por un principio centrado en el tema de la "dinámica no-lineal"; por su parte, Reynoso se centra en la distinción entre los "paradigmas globales" y los "algoritmos", cuyo verdadero principio subyacente es la oposición entre lenguaje natural y lenguaje formal. No obstante, sus esfuerzos, ni Maldonado ni Reynoso problematizan de lleno el meollo de la cuestión: el sentido del concepto ciencia» ${ }^{21}$.

19 Rodríguez Zoyam, L. G., y Rodríguez Zoya, P. G., «El espacio controversial de los sistemas complejos», en: Estudios Filosóficos, no 50 (2014) 110-111.

20 Solana Ruiz, J. L., «El pensamiento complejo de Edgar Morin. Críticas, incomprensiones y revisiones necesarias», en: Gazeta de Antropología, n²7/1 (2011), Artículo 9.

21 Rodríguez Zoya, L. G. y Rodríguez Zoya, P. G., Op. Cit., p. 113. 
Esta cuestión, trasladada al ámbito de las ciencias sociales, se substancia en el debate entre la mayor o menor pertinencia y/o validez de los enfoques cualitativos o cuantitativos para estudiar las dinámicas sociales. Naturalmente, Carlos Reynoso defiende la mayor fiabilidad del enfoque cuantitativo sobre el cualitativo, aunque también sostenga que ambos enfoques son complementarios.

A la hora de abordar esta cuestión fundamental para la filosofía de la ciencia, será inevitable hacer mención de algunas ideas de Reynoso acerca de la complejidad. Pero lo que realmente interesa es esclarecer y criticar, como digo, el concepto más general de lo que es hacer ciencia para Carlos Reynoso.

\section{Planteamiento del problema de la cientificidad en el ámbito de las ciencias DE LA COMPLEJIDAD EN El PENSAMiento de CARLos Reynoso}

Carlos Reynoso reconoce explícitamente que el principal problema cuando se habla de complejidad es que no existe una definición cabal de la misma. Hay un conjunto de disciplinas que estudian fenómenos que se denominan complejos, pero no hay una Teoría General de la Complejidad que permita resolver lo que Karl Popper llamó el problema de la demarcación.

Las disciplinas que abordan problemas denominados complejos son tan dispares como la física, las matemáticas, la termodinámica, la lógica, la sociología, la antropología social, la psicología de corte neurocientífico, la antropoética de Edgar Morin, la teoría de sistemas en sus distintas formulaciones, etc. El significado que cada una de estas disciplinas da a la expresión complejidad es divergente.

Algunos estudiosos de la complejidad, como el profesor Leonardo Rodríguez Zoya, incluso aseguran que las llamadas ciencias de la complejidad, o el mismo pensamiento complejo, no disponen de criterios precisos para identificar a priori un problema como complejo ${ }^{22}$.

El Profesor Carlos Eduardo Maldonado, dela universidad del Rosario(Bogotá), efectivamente reconoce la falta de una Teoría General de la Complejidad. Pero piensa que puede avanzarse en la comprensión y en la delimitación genérica de la misma. Y su mayor mérito, a mi juicio, es intentarlo en el terreno de la filosofía

22 «Ustedes sabrán que la literatura sobre complejidad es enorme, se habla de ciencias de la complejidad, de sistemas complejos, de pensamiento complejo, pero curiosamente no se discute mucho ni se habla tanto, si uno googlea y pone "problemas complejos", nadie habla tanto de problemas complejos, y me parece que es la categoría clave para poder comenzar a discutir los modelos de sociedad y los modelos de futuros deseables (...)». RodRíGuez Zoya, L., ¿Qué tipo de ciencia necesitamos para construir un mundo mejor? Propuesta para el desarrollo de programas de investigación interdisciplinarios en problemas complejos, Castelar: Comunidad Editora Latinoamericana, Buenos Aires 2016, Libro digital, PDF, p. 8. 
de la ciencia (aunque teniendo en cuenta rigurosamente los descubrimientos y aportes metodológicos de las ciencias de la complejidad) ${ }^{23}$.

En la primera parte de su libro Complejidad y Caos, Carlos Reynoso, realiza una exposición descriptiva de las que él considera principales ciencias de la complejidad. Y, a falta de esa Teoría General de la Complejidad, adopta la teoría de la información de Claude Shannon, como marco teórico genérico en torno al cual realizar la taxonomía o clasificación de las ciencias de la complejidad en la actualidad, y su diferencia respecto al pensamiento complejo ${ }^{24}$.

Y lo hace por una razón: porque «(la información) es un factor que es mensurable cuando circula linealmente por un canal; un factor que, cuando se desvinculó de la idea de significado a la que el sentido común se empeña en asociarlo, dio lugar a una teoría que hizo posibles a todas las restantes $»^{25}$. Entre esas otras teorías, Carlos Reynoso incide especialmente sobre dos: la cibernética y la teoría general de sistemas.

Tras dicha elección subyace una convicción básica en Reynoso: la única forma de observar-experimentar con comportamientos complejos es la simulación computacional. Y realizar correctamente dicha simulación, así como comprender sus resultados, requiere de una teoría matemática que describa la naturaleza del procesamiento, compresión y transmisión de información en forma de bits binarios; al tiempo que ayude a reducir el riesgo de que las fuentes de ruido en los canales de transmisión alteren la posibilidad de decodificar la información transmitida. La teoría de la información se ocupa fundamentalmente de esos problemas.

No es extraño, por lo tanto, que Reynoso acepte plenamente los postulados de los programas de investigación del Instituto Santa Fe (basados en la modelización algorítmica de procesos complejos, para experimentar con simulación computacional), y atribuya a las investigaciones de dicho Instituto el mérito de haber precisado el lenguaje y la terminología necesarias para avanzar en la resolución efectiva de problemas complejos en el orden práctico.

Reynoso llega a decir que, con los sistemas de modelización computacional, de carácter algorítmico, se ha avanzado más en la comprensión de la

23 «No existe una teoría general de la complejidad, es cierto. Pero ¿es posible hacer ciencia sin teoría? La pregunta concierne, de manera directa e inequívoca, al ámbito de una teoría y filosofía de la ciencia. En concreto, justamente a una teoría de la complejidad tanto como, simultáneamente, a una filosofía de la ciencia de la complejidad». C. E. Maldonado, «Exploración de una teoría general de la complejidad», en Maldonado, C. E. (Ed.), Complejidad: Revolución Científica y Teoría, Editorial Universidad del Rosario, Bogotá 2009, p. 116.

24 «El concepto de información es parte esencial de la mayor parte de las teorías sistémicas, desde la cibernética hasta los algoritmos genéticos, pasando por la antropología de Bateson, en la que juega un rol decisivo. (...) Como habrá de verse en los capítulos subsiguientes, el concepto de información definido por esa teoría es una referencia inevitable de la mayor parte de las estrategias que guardan alguna relación con la complejidad». Reynoso, C., Complejidad y Caos. Una exploración antropológica, Buenos Aires 2006, p. 34.

25 Reynoso, C., Ibíd., p. 41. 
complejidad en un plazo de diez años que en 30 años de discurso filosófico del pensamiento complejo ${ }^{26}$.

Es más, C. Reynoso está convencido de que las herramientas de modelado computacional suministradas por las nuevas ciencias de la complejidad, surgidas en torno a los postulados y programas de investigación del Instituto Santa Fe, se revelarán suficientemente resolutivas, independientemente del marco teórico en el que se inserten: «Los algoritmos de la complejidad servirán para abordar puntos específicos de la investigación antes que para proporcionar el marco de referencia, el cual podrá articularse en conformidad con la teoría que se quiera ${ }^{27}$. Por ello, dichas herramientas, estarán en condiciones de suministrar elementos de juicio, hipótesis de trabajo y conclusiones relativas a las ciencias de la complejidad y el caos.

Estos elementos serán fundamentales para dar con una Teoría General de la Complejidad o, más sencillamente, para realizar una taxonomía de las ciencias de la complejidad que sea más adecuada a la naturaleza de los problemas complejos.

Es decir, que para Carlos Reynoso las herramientas y métodos de modelado computacional son algo más que un mero utillaje para abordar cuestiones o problemas concretos; tienen también una potencialidad teórica. Pero, eso sí, más enraizada en la naturaleza de los fenómenos complejos que estudian, simulan y -en parte- resuelven.

Por eso es más fiable la teoría que va construyéndose - a cuentagotasa partir de dichas herramientas, que todos los modelos discursivos que han generado un paradigma que ha venido a llamarse "pensamiento complejo», y que no deja de ser un conjunto de afirmaciones genéricas sobre la realidad que usa de forma laxa y anfibológica conceptos extraídos de las ciencias de la complejidad, impidiendo generar algún tipo de propuesta práctica viable a partir de todo su utillaje teórico.

En síntesis, podríamos decir que, para Carlos Reynoso, la ciencia —aquella ciencia que es formalizable - es la única forma de pensamiento fiable. Así que no hay en puridad más que un tipo de ciencia: lo que ha dado en llamarse «ciencia dura» (aquella cuyo lenguaje y base son las matemáticas y la lógica). Este tipo de ciencia observa la realidad mediante un método que permite cuantificar los datos. Una vez cuantificados y formalizados, dichos datos adquieren un significado unívoco, lo que evita las confusiones terminológicas y semánticas propias de las llamadas «ciencias blandas» (las que operan con métodos cualitativos, discursivos — con un tipo de discurso expresado en lenguaje natural o intuitivo- $y$, en definitiva, no cuantificable).

La cuantificación y formalización de las observaciones permiten un tratamiento algorítmico y computacional de las mismas, que es mucho más fiable que la clásica modelización matemática mediante lápiz y papel. Por eso

26 Cfr. Reynoso, C., Ibid., 193.

27 Reynoso, C., Ibíd., pp. 389-390. 
no es de extrañar que, en opinión del profesor Carlos Reynoso, los mayores avances sobre complejidad en los últimos decenios, provengan del campo de la simulación computacional (un tipo de formalización y tratamiento de la información que permite una auténtica interdisciplinariedad en el tratamiento de problemas complejos) ${ }^{28}$.

1.1.- En primer lugar, cabría preguntar cuál es el significado de los conceptos científicos para Carlos Reynoso. ¿Tal vez su definición formal? Por ejemplo, una definición formal del número 2 es «el conjunto de ecuaciones polinómicas que tienen como límite el número 2». Pero esas ecuaciones, lo que hacen es describir la forma o las diferentes formas de acercarnos o de contemplar el número dos. Son distintas perspectivas de dicho número (y nótese cómo ahora, al hablar de «formas de acercarnos o de contemplar», estamos empleando un lenguaje natural basado en la visualización o imaginación o «metáfora», pero lo hacemos estrictamente dentro del campo de la matemática).

Ahora bien, una cosa es describir algo y otra hallar el significado de ese algo (esto es, el sentido, la finalidad, la coherencia interna, y la relación que guarda con el conocimiento humano).

1.2.- En segundo lugar, está la cuestión de si han de ser unívocos los conceptos científicos. Por otra parte, cabría plantearse si es posible la univocidad conceptual en ciencia. Más aún, ¿es posible la univocidad en general? ¿Garantizan los lenguajes formales y cuantitativos dicha univocidad? ¿O bien la inteligencia — la comprensión de los fenómenos- incluye necesariamente un componente hermenéutico, incluso en el ejercicio de las «ciencias duras»?

Por ejemplo, la mecánica cuántica es una de las ramas de la física que está mejor definida desde el punto de vista formal. La descripción del comportamiento de las partículas subatómicas se realiza mediante un conjunto de ecuaciones, cuya fiabilidad se ha comprobado una y otra vez: las ecuaciones de onda de Schrödinger. Sin embargo, hay un debate dentro de la comunidad científica acerca de qué significan realmente dichas ecuaciones. Y existen, al menos, 15 interpretaciones distintas de las mismas ecuaciones.

Podríamos poner el ejemplo de otro lenguaje formal y con pretensión de univocidad: la música. Cada nota es la definición o la representación formal de un tono. Y las notas y símbolos que integran el lenguaje musical son universales, como pueden serlo las matemáticas. Un músico de Nueva York, ante la partitura de la $5^{\mathrm{a}}$ sinfonía de Beethoven, identifica los mismos tonos y ritmos que un músico de Shanghái ante la misma partitura. Y, sin embargo, la música no se ejecuta algorítmicamente, sino que se interpreta. No es lo mismo la interpretación de la $5^{\mathrm{a}}$ sinfonía de Beethoven por la Orquesta Sinfónica de Bilbao bajo la dirección de Solti, que la interpretación de dicha sinfonía por la Filarmónica de Berlín bajo la dirección de von Karajan. Los tonos producidos por los instrumentos son los mismos, pero hay diferencias en la forma de

28 Cf. Reynoso, C., Redes sociales y complejidad. Modelos interdisciplinarios en la gestión sostenible de la sociedad y la cultura, Buenos Aires 2011. 
entender las frases musicales, los tempos, la intensidad...el significado de dicha sinfonía, en fin.

La univocidad se da en el nivel de la definición o descripción (la ecuación), y también en el nivel operacional, pero no en el nivel del significado. Lo cual viene a reforzar la tesis que defendía en el punto anterior: que la definición de un concepto científico no equivale a su significado. Se requiere interpretación. Permítaseme citar aquí un texto del propio Carlos Reynoso; aunque la cita es algo larga, creo que es muy clarificadora:

«Como bien señaló Katherine Hayles en el mejor capítulo de su libro, existen dos tradiciones intelectuales que distintivamente oponen o identifican ambos conceptos: una se remonta a Warren Weaber, el colaborador de Claude Shannon, y la otra a Leon Brillouin; para la primera postura la información se vincula con el desorden y la incertidumbre, para la segunda con el orden y la organización (Wilson 1968; Hyles 1993: 53-86). Se comenta que la decisión de Shannon de llamar entropía a la información le vino de un consejo de John von Neumann, quien le sugirió que usará la palabra porque "nadie sabe qué es la entropía; entonces, en un debate, usted siempre tendrá ventaja" (Denbigh y Denbigh 1985: 104). En una palabra, se sabe perfectamente cómo hacer la medición, pero no existe el menor consenso en cuanto a connotaciones y valores no triviales de lo que se está midiendo: ¿es probabilidad o incertidumbre? ¿Orden o desorganización? Un mensaje bien organizado ¿es más o menos informativo? Convendrá que el lector tenga en cuenta que existen dos formas contrapuestas de interpretar el sentido de la información, ya que cuando se formulen las teorías del caos y la complejidad esta discordancia seguirá dividiendo las aguas. En las humanidades hay una división todavía más drástica entre quienes consideran central el concepto de información (Bateson, el primer Watzlawick) y quienes lo estiman irrelevante o engañoso (Rosen, Maturana, Varela, Morin, Capra) ${ }^{29}$.

Seamos conscientes de que, según esta opinión, la ambigüedad en el significado del concepto de información pone en cuestión la validez de la elección por parte de Carlos Reynoso de la teoría de la información como Teoría General de la complejidad «en funciones», puesto que el sentido del concepto principal de dicha teoría no es claro ni unívoco en el debate científico, según él mismo reconoce. Así pues, la cuestión del significado de los conceptos científicos como algo directamente vinculado a una interpretación que, además es discursiva - filosófica-, queda evidenciada una vez más.

1.3.- En tercer lugar, hay que notar que el debate sobre el significado de los conceptos científicos es un debate genuinamente filosófico y que se realiza discursivamente. Esta era, también, la opinión de Werner Heisenberg. Es cierto que el lenguaje de la filosofía no es un lenguaje "natural», sino de segundo grado (puesto que se pregunta por el significado y el valor de las proposiciones que se formulan en lenguaje natural; esto es, incluye un componente reflexivo y crítico, y además implica el conocimiento de una terminología —más precisa

29 Reynoso, C., Op. Cit., 38-39. 
de lo que piensan algunos científicos— que se adquiere mediante el aprendizaje y el estudio). Sin embargo, para comprender las afirmaciones filosóficas, es necesario poder traducirlas al nivel del lenguaje natural, que opera por analogía y por ideas (imágenes mentales de las realidades).

Así que, por la misma razón, para poder utilizar los conceptos científicos con sentido, o con significado; es decir, para saber qué estamos haciendo cuando realizamos un experimento cualquiera, necesitamos poder explicar lo que hacemos mediante un lenguaje natural. Para ello necesitamos poder visualizar o imaginar o idear de alguna manera lo que expresa una fórmula matemática o un algoritmo. Y ello, a su vez, implica poder comparar esa imagen de dicho concepto o procedimiento con alguna otra imagen de alguna otra realidad que ya $\operatorname{conozcamos}^{30}$.

Los escolásticos decían que la inteligencia opera «per conversionem ad phantasmata», por transformación en imágenes y por comparación entre ellas. Lo expresa lacónicamente Jorge Wagensberg (doctor en física y profesor de teoría de los procesos irreversibles de la Universidad de Barcelona): ¿No se puede comparar?... pues entonces tampoco se puede entender ${ }^{31}$.

1.4.- En cuarto lugar, cabría preguntarse si realmente es más fiable un conocimiento cuantificable que otro de orden cualitativo. Y la respuesta es que puede ser más fiable para ciertas operaciones, pero menos fiable para otras. Si lo que queremos es llevar la contabilidad de una empresa, evidentemente que necesitaremos utilizar conocimientos cuantificables. Pero si lo que queremos es saber por qué hay una determinada partida que, pese a su calidad, siempre acaba generando más gastos que ingresos, habrá que preguntarse por las razones de la falta de demanda. Y ese es un lenguaje cualitativo. Es más, incluso los economistas hablan de la necesidad de incorporar a los balances eso que llaman "intangibles», aunque se dan cuenta de la enorme dificultad que ello conlleva: cuantificar algo cualitativo; algo que sólo puede hacerse, en principio, atribuyendo a una variable un determinado valor por consenso (un consenso alcanzado mediante un debate discursivo y de naturaleza cualitativa).

1.5.- En quinto lugar, habría que matizar que, para contabilizar, o para contar, o para cuantificar, primeramente, hay que realizar una serie de operaciones intelectuales, como ordenar y clasificar, que son obviamente operaciones cualitativas. En un número cualquiera, la ordinalidad tiene prioridad lógica sobre su cardinalidad. Otra cosa es que ambas características son como las dos caras de la misma moneda. No pueden separarse. Pero en esa relación

30 «La construcción de modelos es el arte de elegir aquellos aspectos de un proceso que son importantes para la pregunta que se está haciendo. Como con cualquier arte, esta selección está guiada por el (buen) gusto, la elegancia y la metáfora; es una cuestión de inducción, más que de deducción. La alta ciencia depende de este arte». Holland, J. H., El orden oculto. De cómo la adaptación crea complejidad. FCE, México 1995, p. 158.

31 Wagensberg, J., El pensador intruso. El espíritu interdisciplinario en el mapa del conocimiento, Barcelona 2014, p. 11. 
dialéctica que mantienen cardinalidad y ordinalidad, el principal analogado es el orden, por encima de lo cuantitativo. Ordenar es lo que permite contar.

1.6.- En sexto lugar, Reynoso entiende que el pensamiento complejo o paradigma complejo, está formado por grandes teorías, de naturaleza filosófica y que pretenden erigirse en marcos teóricos generales sobre la noción de complejidad. Estas teorías, debido a su grado de generalización, no son útiles para resolver problemas complejos concretos, puesto que el mismo grado de generalización de aquellas hace que no puedan desarrollar metodologías aplicables y verificables empíricamente. El avance en la resolución de problemas complejos ha venido del desarrollo de una serie de algoritmos o modelos de simulación que permiten experimentar viendo la forma en que evoluciona un determinado sistema al modificar alguna de sus variables iniciales: «Mal que nos pese, la complejidad se resuelve en algoritmos, antes que en teorías. Debido a las razones ya expuestas el algoritmo complejo no admite una inferencia simple que prediga la conducta de lo que él modela. La única forma de averiguar qué puede suceder en escenarios dinámicos es activando el modelo y observando lo que ocurre» ${ }^{32}$. En definitiva: el pensamiento filosófico o los marcos teóricos globales formulados filosóficamente (tales como el pensamiento complejo) no son útiles para la resolución de problemas complejos porque no son traducibles algorítmicamente.

Ante este planteamiento caben algunas objeciones. En primer lugar, reiterar lo que ya decíamos al principio de este epígrafe. Si no sólo queremos operar computacional o algorítmicamente sino saber qué significa lo que estamos haciendo, necesitamos remitirnos al significado del problema que pretendemos resolver mediante el uso de esos algoritmos o modelos. Y dicho significado se alcanza discursivamente, esto es, filosóficamente.

Sólo cuando hemos detectado un problema procedemos a buscar métodos para resolverlo. Pero sólo detectamos un problema cuando observamos un comportamiento que contradice lo que nuestro marco teórico previo (e incluso nuestra cosmovisión) nos indica que es o no posible y probable.

Después necesitamos comprender la naturaleza del problema para poder dar con el método adecuado. Pero no hay un método para encontrar métodos aplicables a cualquier problema. Y, si lo hubiera, podríamos preguntar: ¿y conforme a qué método se construyó el método general de construcción de métodos? Así pues, dar con el método práctico de resolución de un problema depende de la precomprensión teórica que se tenga de ese problema y del debate - discursivo- acerca de cuáles son sus dimensiones más significativas.

Posteriormente se construye un modelo formal que represente el problema en cuestión. Un modelo formal es una red de elementos/conceptos caracterizados por unos axiomas (o definiciones básicas) y cuya interacción está regida por una serie de reglas bien definidas y coherentes. Pero, evidentemente, en el

32 Reynoso, C., op. cit., 389. 
paso del problema real al modelo formal de dicho problema hay un proceso de simplificación y de reducción.

De los casi infinitos aspectos que puede presentar un problema complejo real, el científico opta por algunos que considera más relevantes (por intuición, dado que no hay un método a priori para identificar los aspectos verdaderamente relevantes de un problema dado), por tanto, hay una simplificación. Todo experimento es una simplificación de un fenómeno natural que se quiere observar.

Se simplifican las características (los elementos) del sistema real y también se reduce el conjunto de variables a observar. Eso es lo que hace que el experimento sea factible, o sea, controlable. Y cualquier científico sabe que los resultados que obtenga de la observación experimental (en este caso mediante simulación computacional) son resultados de un modelo simplificado y reductivo.

Pero ese mismo científico también cree que dichos resultados son extrapolables al sistema representado. Porque todo científico cree en el principio de inducción y no sólo en el ejercicio de deducción. Pero el principio de inducción - este es uno de los grandes problemas de la filosofía de la ciencia- no es lógicamente demostrable. Sencillamente se acepta o no se acepta. Se trata de una cuestión filosófica y toda la argumentación que pueda aportarse en este debate es de naturaleza discursiva y cualitativa.

1.7.- Por otro lado, Carlos Reynoso siempre podría argumentar que un científico, ante un problema dado, prescindiendo de marcos teóricos previos, puede construir intuitivamente un modelo formal de dicho problema y comenzar a experimentar siguiendo el esquema what-if (qué pasa si...). Es decir, limitarse a observar las diferencias en la evolución del sistema cuando modifica distintas variables. Esa observación le proporciona información teórica sobre el problema y la acumulación de información mediante el método ensayo-error conducirá precisamente a la formulación teórica que explique el problema o el fenómeno en cuestión. Planteamiento empirista puro.

Este parece ser el enfoque de Carlos Reynoso, porque sostiene que el avance en la comprensión de los fenómenos complejos ha venido de la mano de la construcción de algoritmos que han permitido experimentar mediante simulación computacional:

«La no linealidad y la emergencia hacen que los modelos mecánicos no sean mejores en situaciones de caos; pero las estrategias basadas en la intuición pura (por ejemplo, la hermenéutica) resultan aún más impropias en esos escenarios que el reduccionismo mecanicista. Es por ello que las aproximaciones discursivas a la complejidad, dominadas malgrado suyo por el sentido común, no pueden sacar provecho de las técnicas complejas reales o de las razones que las fundamentan y quedan atrapadas en un juego de analogías convencionales» ${ }^{33}$.

33 Reynoso, C., Complejidad y Caos. Una exploración antropológica, Buenos Aires 2006, p. 387. 
A ello habría que responder que si lo que se quiere es controlar la evolución del fenómeno complejo (para lograr aplicaciones tecnológicas, principalmente), esa puede ser desde luego una táctica que puede funcionar hasta cierto punto. Pero si se quiere comprender (aumentar el conocimiento sobre) su lógica interna, para ello no sirve una estrategia de mera acumulación de conocimientos por ensayo-error. Habrá que organizar esos conocimientos de manera que hagan manifiesta esa lógica interna al fenómeno. Y esa organización no se deriva de la manipulación experimental, sino de un marco teórico previo que ya obra, de hecho, en la mente del científico desde el primer momento en que comenzó a modelizar su sistema formal representativo.

\section{Naturaleza de la simulación Computacional. ¿Desnaturalización del carácter} COMPLEJO DE LA REALIDAD?

Un modelo de simulación computacional es un programa, es decir, un algoritmo o un conjunto de algoritmos diseñados para simular el comportamiento de un determinado sistema. Y ello con la finalidad de comprender algún o algunos aspectos (y resolver problemas) que plantea el funcionamiento de dicho sistema y que remiten a los problemas que emergen del funcionamiento del sistema real formalmente modelado.

Por ejemplo, con el proyecto Millenium Simulation, el Instituto Max Planck de Astrofísica, pudo visualizar y contemplar la estructura tridimensional de un objeto astronómico de tal tamaño que hacía imposible su observación directa como un todo. Los datos observacionales recogidos por varios telescopios durante ocho años fueron tratados informáticamente, incluyendo entre las variables la posible situación de la materia oscura alrededor de las galaxias (en función de las perturbaciones que este tipo de materia origina sobre la rotación y traslación de las galaxias visibles).

Para poder observar dicho objeto - la Gran Muralla Sloan 34 — en su conjunto sería necesario retroceder hasta casi salirse físicamente del mismo universo. Del mismo modo, para advertir su estructura tridimensional, su forma, habría que poder desplazarse alrededor de dicho objeto a una velocidad centenares de miles de veces mayor que la velocidad de la luz. Ambas cosas son imposibles físicamente, pero sí son visualizables a través del computador.

34 La gran muralla Sloan es una pared gigante galáctica en el Universo. Su descubrimiento fue anunciado el 20 de octubre de 2003 por J. Richard Gott III y Mario Jurić, de la Universidad de Princeton, junto con sus colegas, basados en datos del telescopio de exploración digital del espacio Sloan. La pared mide 1.370 millones de años luz de longitud (unos 13.000 trillones de kilómetros) y está situada aproximadamente a mil millones años luz de la Tierra. Para más información, Cf. Springel, White, V. S. D. M., Jenkins, A., Frenk, C. S., Yoshida, N., GaO, L., Navarro, J., Thacker, R., Croton, D., Helly, J., Peacock, J. A., Cole, S., Thomas, P., Couchman, H., Evrard, A., Colberg, J. y Pearce, F. 
El resultado del experimento fue la validación de la hipótesis que sostiene que las galaxias no están distribuidas aleatoriamente por el espacio, sino que forman estructuras a gran escala, cuya forma está determinada por las mismas leyes matemáticas que rigen la formación de la estructura de la espiral de una caracola de mar, o la disposición de las pipas en un girasol, o la estructura de las ramas y las hojas en un árbol cualquiera.

Así pues, la simulación computacional realmente es una herramienta experimentalmente válida, y que puede arrojar luz y una nueva comprensión sobre determinados marcos teóricos. Tal y como defiende Carlos Reynoso, es más que un mero utillaje tecnológico, y posee una virtualidad teórica. La filosofía de la ciencia, de hecho, siempre ha insistido en la mutua interdependencia de teoría y experimentación.

Sin embargo, el ejercicio de reducción y simplificación que implica cualquier experimento también es imputable a la modelización computacional entendida como experimento. Si una de las características que define la complejidad es la no reductibilidad del todo a la suma de sus partes ¿no significa eso que la modelización computacional de un sistema complejo desvirtúa a la fuerza su carácter complejo, reduciéndolo a una «complejidad observable y controlable» (que equivale a una no-complejidad)?

La respuesta de Carlos Reynoso a esta cuestión es que la complejidad es un efecto del abordaje aplicado a los objetos de estudio concebidos bajo el influjo de un modelo, no una propiedad ontológica intrínseca de aquello que se analiza:

«La escala de un modelo respecto de lo real es indecidible, ya que la realidad puede ser casi infinitamente descompuesta, es analíticamente inagotable; una ameba puede llegar a ser tan complicada como un sistema planetario; el sol y la tierra, con ser inmensos y albergar tantas cosas, constituyen un sistema "pequeño", pues astronómicamente tienen sólo doce grados de libertad. No hay entonces una escala propia de los fenómenos: teorías que tratan de enormes conjuntos sociales son a menudo más sencillas que teorías que abordan la personalidad de sujetos individuales» ${ }^{35}$.

Esto sencillamente no es cierto, pues la ontología —la cosmovisión necesaria para entender un problema y buscar intuitivamente un método adecuadosiempre precede al modelo. Lo cual quiere decir que la complejidad fruto del modelo no es equiparable a la complejidad del sistema real modelado; complejidad que es real y existente objetivamente, y que hace referencia no tanto al número de elementos de que consta un sistema, cuanto a la estructura en red en la que estos elementos se hallan interconectados formando un todo orgánico.

Es esa estructura y su funcionamiento lo que se quiere entender mediante la simulación computacional (o ejecución algorítmica de un modelo) de dicha estructura. Y es cierto que un algoritmo en apariencia simple o de muy pocos elementos puede generar propiedades emergentes que pueden considerarse

35 Reynoso, C., Complejidad y Caos. Una exploración antropológica, Buenos Aires 2006. p. 14 . 
como complejas. Pero, insisto, la complejidad generada computacionalmente no es parangonable con la complejidad de una ameba o a la complejidad del «problema de los tres cuerpos» (H. Poincaré).

Es cierto que no hay percepción sin interpretación; y que no hay interpretación sin teoría. En ese sentido, la complejidad es una forma de mirar la realidad desde una teoría (la no-linealidad de muchos fenómenos naturales y el carácter evolutivo y abierto de la propia naturaleza). También en ese sentido la complejidad es propia del fenómeno y no una característica de la realidad considerada en-sí misma. Pero es que lo que denominamos realidad fenoménica es la realidad observable y observada. Por tanto, podemos afirmar sin ambages que la complejidad es una característica de la realidad, tal y como - fenoménicamente- es percibida por nosotros.

Ahora bien, lo que no es cierto es que la complejidad sea resultado del abordaje de un problema bajo el influjo de un modelo. El sistema formal (modelo) pretende reflejar de alguna manera la dinámica real que observamos en el fenómeno.

Por ello, la naturaleza de la complejidad simulada mediante modelos formales computacionales cambia según la escala de observación y análisis que apliquemos. Para construir el modelo hemos tenido que seleccionar elementos, estructuras de conexión y variables, y prescindir de otras; es decir, hemos tenido que situarnos en una escala de observación determinada. Pero esa escala de observación no es «la propia del objeto», porque cualquier objeto admite infinitas escalas de observación y/o aproximación.

La escala de observación que hemos adoptado viene determinada por la estructura del acto de observación y por las herramientas y convenciones con las que vamos a efectuar las mediciones y la cuantificación de los datos. Por ello, es cierto que la escala de observación de un objeto es indecidible metodológicamente, tal y como afirma Reynoso. La escala de observación incluye siempre un carácter de arbitrariedad (como la aplicación de los métodos o como los actos de medición). Pero ello no significa que no exista realmente el objeto observado - mediante la técnica de simulación computacional—, ni tampoco que dicho objeto no posea, como cualidad intrínseca, complejidad.

Podría decirse que la diferencia de la complejidad simulada (que es verdadera complejidad) respecto a la complejidad del objeto real simulado (que también es verdadera complejidad) radica en que la emergencia de propiedades sistémicas en el proceso de simulación es de carácter epifenoménico, mientras que la emergencia de propiedades sistémicas en los sistemas biológicos, físicos, etc., es de carácter substancial. Aquello que emerge en la naturaleza cobra entidad propia, no así en la simulación computacional.

Y para probar que esto es así, basta con observar, no ya la evolución de la vida, que ha generado una increíble biodiversidad, con sistemas orgánicos autónomos e independientes de aquellos de los que proceden, sino la misma evolución de la materia que, a partir del hidrógeno, ha generado todo el conjunto de elementos que conforman la tabla periódica y cuyas propiedades son irreductibles ontológicamente y ónticamente a los elementos que les preceden. 
3. SiMULACIÓN COMPUTACIONAL: ¿NUEVA VERSIÓN DE UN EXPERIMENTO MENTAL?

Un experimento mental consiste en imaginar un escenario hipotético construido en base a ciertas normas que nos permitan también imaginar las relaciones y los procesos que tendrían lugar entre ciertos objetos (los objetos que estamos estudiando) con el fin de comprender mejor su naturaleza y afinar así nuestra argumentación.

Ernst Mach empleó el término experimento mental de una manera distinta. Para él era la construcción imaginaria de un experimento que tendría que poder ser llevado posteriormente a la práctica; es decir, el proceso de modelado de un experimento físico real. Pero en el campo de los problemas complejos rara vez pueden diseñarse modelos teóricos que puedan experimentarse de forma directa en la realidad física. Por eso se utilizan los métodos de simulación computacional.

En este sentido, un experimento de simulación computacional referente, por ejemplo, a la evolución de la distribución del cableado telefónico de una ciudad nos ahorra el esfuerzo de imaginar cómo sería dicha evolución a partir de unos parámetros iniciales y reglas (entre otras cosas porque la limitación cognitiva de nuestra mente para tratar con datos cuantitativos muy abundantes - que pueden proceder de escasos elementos, cuidado- nos imposibilita imaginarla adecuadamente), y nos permite observarlo, paso a paso, en una pantalla.

Pero lo que observamos no es algo meramente objetivo. Observamos una representación en imágenes construidas a partir de unas condiciones hipotéticas también imaginarias. Digamos que el experimento de simulación computacional es una herramienta que amplifica nuestra capacidad de imaginar escenarios alternativos posibilitándonos incidir sobre problemas reales. En ese sentido, podría considerarse un experimento mental.

Y esto es muy notable, como decía, porque un experimento mental es de naturaleza discursiva y metafórica. Basta con pensar en el experimento mental del Demonio de Maxwell, o del Gato de Schrödinger. Pues bien, la naturaleza discursiva y metafórica (o imaginativa) de dicho experimento se mantiene aunque para realizarlo utilicemos una herramienta computacional de apoyo.

\section{CONCLUSIÓn: ¿ModelizACIÓN DEL PENSAMIENTO DISCURSIVo?}

Si las observaciones precedentes son correctas, resulta que: las formulaciones algorítmicas de los modelos computacionales no tienen un significado unívoco (sí una definición y un uso); que dichas formulaciones son altamente simplificadoras y selectivas (no puede ser de otro modo si se quiere que sean operativas); que dicha selección y reducción se realiza intuitivamente por el científico; y que la simulación computacional obtenida es la prolongación o la ampliación de un experimento mental (imaginativo y discursivo).

¿Cómo seguir sosteniendo, entonces, tal y como hace Carlos Reynoso, que el pensamiento complejo no es válido como marco teórico porque no es aplicable a la construcción de algoritmos, que es lo que realmente resuelve los problemas 
prácticos? Da la impresión de que Carlos Reynoso entiende la ciencia más por analogía a la ingeniería que por analogía a la física o las matemáticas.

En sus numerosas críticas al pensamiento complejo de Edgar Morin, Henri Atlan, Ilia Prigogine, Humberto Maturana y Francisco Varela, Carlos Reynoso dice, de manera reiterativa, que estos autores - especialmente Morin- han detenido arbitrariamente sus investigaciones sobre complejidad en la década de los 80-90 del pasado siglo. Y que, precisamente a partir de esas fechas es cuando surgen los avances en la comprensión de la complejidad gracias, entre otras cosas, a los trabajos del Instituto Santa Fe (programas de modelización computacional para la simulación de sistemas adaptativos complejos, entre otro tipo de algoritmos que Reynoso menciona).

Esta crítica es aceptable, aunque ese déficit es comprensible puesto que nadie está en condiciones de abarcar y comprender todas las nuevas disciplinas que van surgiendo, ni - mucho menos- estar al tanto de todas las publicaciones respecto a un área tan extensa como la de las ciencias de la complejidad.

Pues bien, recientemente se defendió y publicó una tesis doctoral muy relevante para nuestro debate: la del profesor Leonardo Rodríguez Zoya, en la que éste demuestra que no sólo existe complementariedad entre modelos cualitativos y modelos cuantitativos, sino que existe incluso una permeabilidad o algún tipo de continuidad entre ambos.

La tesis doctoral del profesor Zoya demuestra que el modelo epistemológico del pensamiento complejo posee unos principios teóricos y metodológicos que lo hacen susceptible de ser empleado en una investigación epistemológica de carácter empírico.

Lo curioso es que el objeto de estudio que escogió Leonardo Rodríguez Zoya para la elaboración de su tesis doctoral fue el estudio crítico de la construcción de conocimiento en la comunidad de investigadores que trabajan en sistemas complejos y simulación social.

Pues bien, la motivación que guía este monumental trabajo es doble. Por un lado, fundamentar una metodología de investigación epistemológica de carácter interdisciplinario, empírico y crítico. Y, en segundo lugar, afrontar «el desafío de articular críticamente dos tradiciones de investigación con escasos puntos de contacto entre sí: el enfoque del pensamiento complejo desarrollado por Edgar Morin y el de las ciencias de los sistemas complejos desarrolladas en el campo anglosajón y europeo-continental (Maldonado, 1999; Morin, 2007; Reynoso, 2009). El diseño metodológico de la investigación empírica plantea un estudio psicosocial de las creencias científicas mediante la articulación complementaria de métodos cualitativos y cuantitativos, en particular, el análisis cualitativo del discurso científico y el empleo de técnicas estadísticas y psicométricas» ${ }^{36}$.

Rodríguez Zoya, siguiendo el pensamiento interdisciplinario de Rolando

36 Rodríguez Zoya, L. y Roggero, P., «El modelo epistemológico del pensamiento complejo. Resultados de una investigación empírica y crítica en sistemas complejos y lineamientos para un programa de investigación en epistemología compleja», en: $X X I X$ Congreso Latinoamericano de Sociología, Santiago de Chile, 2013, p. 2. 
García, distingue entre varias clases de modelos que pueden ser articulados. En primer lugar, los modelos mentales que generalmente son implícitos. Aquí podrían incluirse las ideas o expectativas que cada científico tiene sobre su propia área; o bien lo que denominamos cosmovisiones. Estos modelos mentales son de carácter individual pero fundamentan nuestra capacidad de diálogo y contraste intersubjetivo.

Cuando compartimos nuestros modelos mentales con la finalidad de crear un pensamiento común, transformamos dichos modelos en modelos discursivos; que son modelos expuestos en lenguaje natural. Naturalmente que los modelos formulados en lenguaje natural son ambiguos, dado que su inteligibilidad depende de los múltiples contextos de interpretación. Por ello necesitamos traducir los modelos discursivos a modelos conceptuales. Y esto implica precisar el significado de los conceptos que estamos utilizando de una forma sistemática, es decir, metódica.

Cuando alcanzamos el nivel conceptual y sistemático en nuestro discurso, estamos en condiciones de traducir tales conceptos que empleamos, en un sistema que puede ser formalizado. Así se construyen los modelos matemáticos y lógicos.

Finalmente, según el pensamiento del Profesor Leonardo Rodríguez Zoya, obtendremos los modelos operacionales u algorítmicos, que son los programas ejecutables en una computadora.

Pero necesitamos una herramienta que nos permita traducir el significado de unos modelos a otros. Rodríguez Zoya propone como herramienta a utilizar en dicha traducción el lenguaje unificado de modelado (UML). Este lenguaje es un conjunto de símbolos que pueden vincularse mediante diagramas gráficos con conectores lógicos. La utilización de esta herramienta permite la discusión y el intercambio de distintos significados conceptuales entre especialistas, y llegar a puntos de acuerdo que permitan eliminar ciertas ambigüedades conceptuales. Se trata de una herramienta para construir pensamiento común.

A partir de los mapas o redes conceptuales sistemáticas generadas utilizando el lenguaje UML, los especialistas en programación pueden traducir o representar esa red conceptual mediante un lenguaje de programación, precisamente porque los conceptos de dicho mapa están vinculados entre sí por conectores lógicos.

Esta herramienta fue utilizada y probada con éxito en la tesis doctoral mencionada. Así pues, los esfuerzos discursivos de carácter filosófico, cuya finalidad es pensar con rigor acerca de la complejidad, comprenderla y crear un marco epistemológico general, no están reñidos con los esfuerzos por modelar algorítmicamente experimentos de simulación computacional sobre complejidad. Con lo que la presunta oposición entre pensamiento complejo y algoritmos, sostenida por Carlos Reynoso, se revela como falsa.

Por otro lado, una de las facetas más relevantes de la tesis doctoral de Leonardo Rodríguez Zoya es que no sólo aborda el carácter complejo en que una ciencia es conceptualizada, sino que se detiene a analizar el sistema de creencias científicas: 
«La teoría del sistema de creencias científicas pretende dar cuenta de la configuración de patrones organizativos de pensamiento y acción. El concepto de creencia científica alude a una forma de cognición social, es decir, construcciones sociales de carácter cognitivo y construcciones cognitivas de carácter social. En otros términos, las creencias científicas son un conjunto organizado de "representaciones mentales socialmente compartidas» ${ }^{37}$.

Esta constatación, por supuesto, falsa la «creencia científica» de C. Reynoso de que todo lo que se hace en ciencia conforme a una metodología formalizadora es plenamente objetivo y, por ello, la única fuente fiable de teorización. No hay teorización sin representación mental. Y no hay representación mental sin un ejercicio de comunicación intersubjetivo de dichas representaciones, que se produce necesariamente en un plano discursivo y en lenguaje natural.

En definitiva, creo que lo que hace a Carlos Reynoso decantarse por el modelo algorítmico como único modelo válido para entender la complejidad es una deficiente concepción de la ciencia experimental. En alguna ocasión, Reynoso indica que la formalización hace posible las operaciones sin una referencia semántica (que según él es lo que corrompe el discurso científico al introducirse el lenguaje natural).

Algo parecido a lo que dijo Isaac Newton al ser preguntado por la hipótesis del éter. "Hipothesis non fingo», contestó lacónicamente (lo que se demostró lamentablemente falso tras su muerte, al encontrarse numerosos documentos en los que el genio inglés especulaba y reflexionaba sobre la posibilidad del éter). Esta noción de la ciencia experimental como directamente derivada de la observación, sin la mediación de hipótesis, teorías y cosmovisiones previas es rotundamente falsa. No observamos nada si no tenemos una idea acerca de qué debemos observar. Y esa idea previa es la hipótesis de trabajo, que necesariamente será representada imaginativamente y formulada discursivamente en diálogo con otros científicos.

El problema principal para Carlos Reynoso y para todos los que tienen una ideología de tipo cientificista consiste en aceptar que el científico, en un determinado momento, saltará del registro observacional al registro interpretativo. Y en ese mismo instante estará utilizando la filosofía como mediación hermenéutica del dato cuantificable; y el lenguaje discursivo, como mediación de la analogía (que se produce como comunicación intersubjetiva).

Universidad de Deusto. Bilbao

mrviguri@deusto.es
Miguel Ramón Viguri Axpe

[Artículo aprobado para publicación en enero de 2018]

37 Rodríguez Zoya, L. G. y Roggero, P., Ibíd., p. 5 